\title{
Star formation and dust extinction properties of local galaxies from the AKARI-GALEX all-sky surveys
}

\section{First results from the most secure multiband sample from the far-ultraviolet to the far-infrared}

\author{
T. T. Takeuchi ${ }^{1}$, V. Buat ${ }^{2}$, S. Heinis ${ }^{3}$, E. Giovannoli ${ }^{2}$, F.-T. Yuan ${ }^{4}$, \\ J. Iglesias-Páramo ${ }^{5}$, K. L. Murata ${ }^{4}$, and D. Burgarella ${ }^{2}$ \\ 1 Institute for Advanced Research, Nagoya University, Furo-cho, Chikusa-ku, Nagoya 464-8601, Japan \\ e-mail: takeuchi@iar.nagoya-u.ac.jp \\ 2 Laboratoire d'Astrophysique de Marseille, OAMP, Université Aix-Marseille, CNRS, 38 rue Frédéric Joliot-Curie, \\ 13388 Marseille Cedex 13, France \\ e-mail: [veronique.buat; elodie.giovannoli; denis.burgarella]@oamp.fr \\ 3 Department of Physics \& Astronomy, The Johns Hopkins University, 3701 San Martin Drive, Baltimore, MD 21218, USA \\ e-mail: sebastien@pha.jhu.edu \\ 4 Division of Particle and Astrophysical Science, Nagoya University, Furo-cho, Chikusa-ku, Nagoya 464-8602, Japan \\ e-mail: [yuan. fangting; murata.katsuhiro]@g.mbox.nagoya-u.ac.jp \\ 5 Instituto de Astrofísica de Andalucía (IAA, CSIC), Camino Bajo de Huétor 50, 18008 Granada, Spain \\ e-mail: jiglesia@iaa.es
}

Received 15 October 2009 / Accepted 21 December 2009

\section{ABSTRACT}

\begin{abstract}
Aims. We explore spectral energy distributions (SEDs), star formation (SF), and dust extinction properties of galaxies in the Local Universe.

Methods. The AKARI all-sky survey provided the first bright point source catalog detected at $90 \mu \mathrm{m}$. Beginning with this catalog, we selected galaxies by matching the AKARI sources with those in the IRAS point source catalog redshift survey. We measured the total GALEX FUV and NUV flux densities with a photometry software we specifically developed for this purpose. In a further step we matched this sample with the Sloan digital sky survey (SDSS) and 2 micron all sky survey (2MASS) galaxies. With this procedure we obtained a basic sample which consists of 776 galaxies. After removing objects whose photometry was contaminated by foreground sources (mainly in the SDSS), we defined the "secure sample" which contains 607 galaxies.

Results. The sample galaxies have redshifts of $\lesssim 0.15$, and their $90-\mu$ m luminosities range from $10^{6}$ to $10^{12} L_{\odot}$, with a peak at $10^{10} L_{\odot}$. The SEDs display a large variety, especially more than four orders of magnitude at the mid-far-infrared (M-FIR), but if we sort the sample with respect to $90 \mu \mathrm{m}$, the average SED shows a coherent trend: the more luminous an SED at $90 \mu \mathrm{m}$, the redder the global SED becomes. The $M_{r}-\mathrm{NUV}-r$ color-magnitude relation of our sample does not show bimodality, and the distribution is centered on the green valley. We established formulae to convert the FIR luminosity from the AKARI bands to the total IR (TIR) luminosity $L_{\text {TIR }}$. The luminosity related to the SF activity $\left(L_{\mathrm{SF}}\right)$ is dominated by $L_{\mathrm{TIR}}$ even if we take into account the FIR emission from dust heated by old stars. At a high SF rate (SFR) $\left(>20 M_{\odot} \mathrm{yr}^{-1}\right)$, the fraction of the directly visible SFR, SFR FUv, decreases. We also estimated the FUV attenuation $A_{\mathrm{FUV}}$ from the FUV-to-TIR luminosity ratio. We examined the $L_{\mathrm{TIR}} / L_{\mathrm{FUV}}$-UV slope (FUV - NUV) relation. The majority of the sample has $L_{\mathrm{TIR}} / L_{\mathrm{FUV}}$ ratios five to ten times lower than expected from the local starburst relation, while some luminous IR and all the ultraluminous IR galaxies of this sample have higher $L_{\mathrm{TIR}} / L_{\mathrm{FUV}}$ ratios. We found the attenuation indicator $L_{\mathrm{TIR}} / L_{\mathrm{FUV}}$ correlated to the stellar mass of galaxies, $M_{*}$, but not with a specific SFR, SFR/ $M_{*}$.

Conclusions. Together, these results show that the AKARI survey gives a representative sample of the local SF galaxies which will be a comprehensive local standard of their various properties to be compared with, for instance, high- $z$ SF galaxies.
\end{abstract}

Key words. dust, extinction - infrared: galaxies - ultraviolet: galaxies - galaxies: evolution - galaxies: starburst - stars: formation

\section{Introduction}

Star formation history of galaxies is one of the most important and exciting topics in extragalactic astrophysics and observational cosmology. Especially exploring the "true" absolute value of the cosmic star formation rate (SFR) has been of a central importance.

However, it remained difficult for a long time because of dust extinction. Even in the Local Universe, there are some problems to overcome to estimate the absolute value of the SFR density because of a different dependence of various SFR estimators on dust attenuation (e.g. Hopkins \& Beacom 2006, and references therein). Active star formation (SF) always comes along with dust production because of various dust grain formation processes related to the final stage of stellar evolution (e.g. Dwek \& Scalo 1980; Dwek 1998; Nozawa et al. 2003; Takeuchi et al. 2005c). Observationally the SFR of galaxies is measured by the ultraviolet (UV) luminosity of massive stars because of their short lifetime $\left(\sim 10^{8} \mathrm{yr}\right)$ compared with the age of galaxies or the Universe. However, the UV photons are easily scattered 
and absorbed by dust grains. Hence the SFR of galaxies is always inevitably affected by dust which is produced by their SF activity. Since the absorbed energy is re-emitted at farinfrared (FIR) wavelengths, it is essential to observe galaxies both at the UV and the FIR to have an unbiased view of their SF (e.g. Buat et al. 2005; Seibert et al. 2005; Cortese et al. 2006; Takeuchi et al. 2005a).

To know the history of the SFR in the Universe, we must know the starting reference value, i.e. the SFR density in the Local Universe: otherwise we cannot define how much larger the SFR was in the past. However, since the volume of the Local Universe is limited by definition, an all-sky survey is the only viable way to refine our knowledge of local galaxies. On the "directly visible SF" side the advent of the UV surveyor-type satellite GALEX (Martin et al. 2005) has changed the situation of UV astronomy drastically. GALEX is performing an all-sky survey (all sky imaging survey: AIS) at FUV (1530 ̊) and NUV (2300 $\AA$ ) with detection limits of 19.9 and 20.8 mag (Morrissey et al. 2007), as well as deep surveys in some selected regions. In a previous study we showed that GALEX AIS provides us with an unprecedented opportunity to explore the visible SF in the Local Universe (Buat et al. 2007a).

As for the "hidden" side of SF, IRAS (Neugebauer et al. 1984) has brought a vast amount of statistics of dusty galaxies in the Local Universe in the IRAS point source catalog (PSC) (see e.g. Soifer et al. 1987). Subsequently FIR facilities with much higher sensitivity have been launched, like ISO (e.g. Genzel \& Cesarsky 2000; Verma et al. 2005) and Spitzer (e.g. Soifer et al. 2008), and revealed the deep IR universe, but the latter two were observatories dedicated to pointed observations.

In contrast to the latter two facilities, the Japanese IR satellite AKARI has performed various large-area surveys at IR wavelengths (Murakami et al. 2007) after IRAS, especially including all-sky surveys at the FIR and MIR. In particular, with the aid of the far-infrared surveyor (FIS: Kawada et al. 2007) onboard, various IR surveys were performed by AKARI. AKARI FIS has four FIR wavebands centered on 65, 90, 140, and $160 \mu \mathrm{m}$, and a FIR all-sky survey was completed by this instrument. Since the latter two bands are longer than $100 \mu \mathrm{m}$, which was the longest wavelength band of IRAS, the obtained sample of dusty galaxies is less biased than the IRAS sample, i.e. thanks to the better sensitivity to cooler dust than IRAS, AKARI can detect galaxies which emit dust continuum radiation with lower temperatures. In addition to these SF-related wavelengths, we need other various wavelength bands to examine the physical properties of galaxies, as for instance stellar mass closely related to near-IR (NIR) and intermediate stellar population related to optical. For the former we have a set of all-sky data from the 2-micron allsky survey (2MASS: Skrutskie et al. 2006), and for the latter, the SDSS final data (DR7) are publicly available ${ }^{1}$, even if SDSS is not an all-sky survey.

We constructed a multiband galaxy catalog based on the AKARI all-sky survey $90-\mu \mathrm{m}$ selected sources associated with IRAS PSC $z$ galaxies (Saunders et al. 2000). Then we measured the GALEX FUV and NUV flux densities and associated SDSS and 2MASS photometries. For this initial study, we have only selected "secure" galaxies with good photometric measurements for most of the bands. We present the sample construction in Sect. 2. We describe the basic properties of galaxies in the sample in Sect. 3. Results on the SF and dust attenuation are presented in Sect. 4. Section 5 is devoted to our summary and conclusions.

\footnotetext{
1 URL: http://www.sdss.org/dr7/
}

Throughout the paper we will assume $\Omega_{\mathrm{M} 0}=0.3, \Omega_{\Lambda 0}=$ 0.7 and $H_{0}=70 \mathrm{~km} \mathrm{~s}^{-1} \mathrm{Mpc}^{-1}$. The luminosities are defined as $v L_{v}$ and are expressed in solar units assuming $L_{\odot}=$ $3.83 \times 10^{33} \mathrm{erg} \mathrm{s}^{-1}$.

\section{Sample construction}

\subsection{Construction of the parent FIR sample from AKARI and IRAS PSCZ}

\subsubsection{AKARI FIS all-sky survey}

The primary purpose of the AKARI mission is to provide second-generation infrared (IR) catalogs with a better spatial resolution and wider spectral coverage than the IRAS catalog. AKARI is equipped with a cryogenically cooled telescope with an aperture diameter of $68.5 \mathrm{~cm}$ and two scientific instruments, the FIS (Kawada et al. 2007) and the infrared camera (IRC; Onaka et al. 2007). Among various astronomical observations performed by AKARI, an all sky survey with the FIS has been carried out (AKARI all-sky survey). Since the FIS is an instrument dedicated to the FIR $\lambda=$ 50-180 $\mu \mathrm{m}$, all the AKARI FIS bands are in the FIR wavelengths: N60 $(65 \mu \mathrm{m}), W I D E-S(90 \mu \mathrm{m}), W I D E-L(140 \mu \mathrm{m})$, and $N 160(160 \mu \mathrm{m})$ (Kawada et al. 2007). Hereafter we note as $S_{65}$, $S_{90}, S_{140}$ and $S_{160}$ the flux densities in these bands. Especially as the FIS has sensitivity at longer wavelengths than the detector of IRAS, a new classification scheme is needed if we try to select a certain class of objects. Such a scheme is not merely an empirical technique but will also provide us with a new understanding of objects with cool dust which were difficult to detect by the IRAS bands.

We use the AKARI FIS bright source catalogue (BSC), the first primary catalog from the AKARI all-sky survey. Data from the $\beta$ - 1 version of this catalog are used in this work. AKARI BSC is supposed to have a uniform detection limit corresponding to per scan sensitivity over the entire sky, except for very bright sky parts where a different data acquisition mode had to be applied. A summary of the all-sky survey is presented in Yamamura et al. (2009). The AKARI FIS BSC provides data for 64311 sources. For each detected source the AKARI source identifier, the equatorial coordinates of the source position and the flux densities in the four FIR bands are given. Errors are not estimated for each individual source, but instead they are estimated in total to be $35 \%, 30 \%, 60 \%$, and $60 \%$ at N60, WIDE-S, WIDE-L, and N160, respectively (Yamamura et al. 2008). The AKARI IRC performed another all sky survey, but the data were not fully reduced at the time we prepared this paper. Hence we focus on the FIS data only.

\subsubsection{Matching with IRAS PSCz}

The AKARI BSC contains many Galactic sources, like AGBs, HII regions, planetary nebulae, etc. (e.g. Pollo et al. 2010). In order to construct a reliable catalog of galaxies, we should pick up the FIS sources confirmed as galaxies. For this purpose we have performed a cross identification of the BSC sources with the IRAS PSC $z$ (Saunders et al. 2000). The PSC $z$ is a redshift survey of galaxies selected at the IRAS $60 \mu \mathrm{m}$ with a flux density limit of $S_{60}>0.6[\mathrm{Jy}]^{2}$. The PSC $z$ contains $\sim 16000$ galaxies. We put a limit on the recession velocity $v>1000 \mathrm{~km} \mathrm{~s}^{-1}$

\footnotetext{
2 Because of this step we note that we do not make maximal use of the advantage of the long wavelength bands of the AKARI FIS, since the sample is limited by the IRAS bands $(\lambda<100 \mu \mathrm{m})$.
} 
so we could avoid the effect of the peculiar velocity of galaxies. As a next step we matched the AKARI BSC sources with the $\mathrm{PSC} z$ galaxies with a search radius of 36 arcsec, which corresponds to the position uncertainty of the IRAS PSC. The number of matched sources was 5890. To examine the effect of the choice of our search radius, we changed the criterion from 20 to 60 arcsec. This change of radius did not have a significant impact on the resulting catalog $(\$ 5 \%)$. Hence we used the catalog with a search radius of 36 arcsec in the analysis below.

To broaden our analysis we even further made a cross identification with the SDSS galaxies (see Sect. 2.3). Then we restricted our data to only the area covered by the SDSS DR7, which is $8378.015 \mathrm{deg}^{2}$.

The restriction to this region of the sky has the advantage that the Galactic diffuse FIR emission is strong in some areas of the sky, and consequently the FIR flux densities of point sources measured there are contaminated by the Galactic emission and not very accurate. As the SDSS region is selected in a way to have only a faint Galactic extinction, the selected area automatically avoids these FIR-bright regions. The resulting parent cata$\log$ contains 1186 galaxies.

\subsubsection{Flux density comparison between AKARI and IRAS}

We compared the AKARI and IRAS flux densities to examine our sample selection. The correlation is shown in Fig. 1. The horizontal dotted lines in the upper-left, upper-right, and lowerleft panels represent the flux density limit of the IRAS PSC $z$. The AKARI BSC sources are selected at WIDE-S, i.e. $90 \mu \mathrm{m}$. It is important to see which selection controls the sample selection. As seen in the upper-left panel, both the IRAS PSC $z$ and AKARI limit are well-defined, and neither of them strongly restricts the sample. The effective $90 \mu \mathrm{m}$ flux density limit of our parent sample is $\sim 0.8 \mathrm{Jy}$.

\subsection{GALEX photometry}

GALEX AIS now observed $25000 \mathrm{deg}^{2}$ at FUV and NUV. The latest version of the public imaging is GR4/GR5 $5^{3}$. We have measured the FUV and NUV photometry of the parent AKARI galaxies as follows:

1. Cut out a $30^{\prime} \times 30^{\prime}$ square subimage from the GALEX AIS images around each AKARI galaxy.

2. Select a subimage with the largest exposure time when multiple observations were available.

3. Measure the FUV and NUV flux densities. The NUV observation is taken as our reference.

Since the sky coverage of GALEX AIS is not complete, we do not have a proper GALEX image for an AKARI galaxy in some cases. In this case we excluded the galaxy because we did not have any UV information.

Almost all of the sources are resolved by GALEX. They are thus very often separated into small bright patchy regions, and the GALEX pipeline misidentifies these fragments as individual objects. This is referred to as shredding. We must deal with the shredding to obtain sensible flux density measurements for nearby extended galaxies. For this purpose we specifically developed an IDL software package. This software performs aperture photometry in the NUV sub-image using a set of elliptical apertures. Total flux density is calculated within the aperture corresponding to the convergence of the growth curve. The sky

${ }^{3}$ URL: http://galex.stsci.edu/GR4/
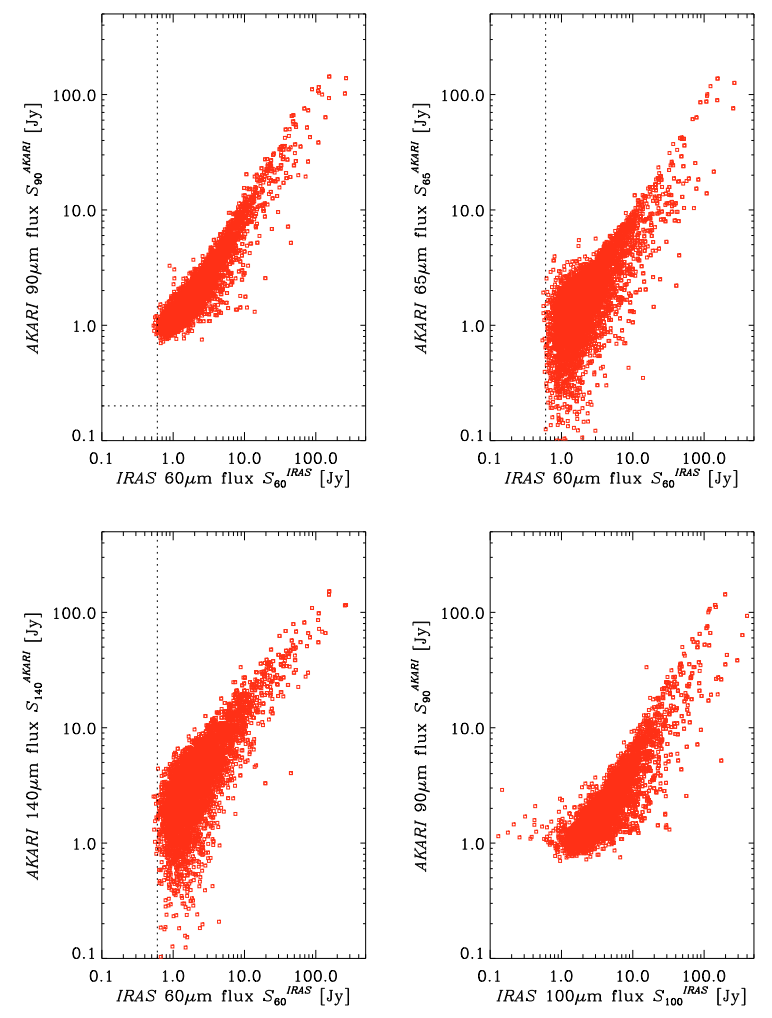

Fig. 1. Comparison between the AKARI FIS and IRAS PSC $z$ flux densities. Upper-left, upper-right, and lower-left panels present comparisons of the IRAS $60 \mu \mathrm{m}$ with AKARI $90 \mu \mathrm{m}, 65 \mu \mathrm{m}$, and $140 \mu \mathrm{m}$ flux densities of the AKARI-IRAS correlated sample. The vertical dotted lines in these panels represent the flux density limit of the IRAS PSC $z$. Lowerright panel shows a comparison of the IRAS $100 \mu \mathrm{m}$ with the AKARI $90 \mu \mathrm{m}$ flux densities. The horizontal dotted line in the upper-left panel represents the formally expected detection limit of the FIS $90 \mu \mathrm{m}$.

background is measured by combining several individual regions around the source. The NUV and FUV flux densities are corrected for Galactic extinction using the Schlegel map (Schlegel et al. 1998) and the Galactic extinction curve of Cardelli et al. (1989). A detailed description of the photometry process can be found in Iglesias-Páramo et al. (2006).

This software was already used for our previous IRASGALEX based studies (Buat et al. 2007a), and its performance is carefully checked and established. During the procedure we also excluded all the sources that were either contaminated by stars or too close to be disentangled by the photometry software. After this procedure the number of remaining galaxies is 776 .

\subsection{Matching with the SDSS and 2MASS galaxies}

As a next step we matched the AKARI-IRAS PSC $z$-GALEX sample (776 galaxies, hereafter abbreviated as the AKARIGALEX sample) with the SDSS and 2MASS.

The AKARI-GALEX sample was matched to the 2MASS all-sky Extended Source (XSC) catalog to obtain the NIR ( $J$, $H$, and $K \mathrm{~s}$ ) flux densities. A matching radius of $20^{\prime \prime}$ was initially adopted for the cross correlation. All but seven out of the 776 galaxies in the AKARI-GALEX sample showed 2MASS counterparts at distances closer than the matching radius. For multiple candidates we always selected the brightest one at $K$ s-band as the most plausible one. A further cross correlation with a matching radius of $30^{\prime \prime}$ was attempted for these seven galaxies with no $2 \mathrm{MASS}$ counterpart within the 


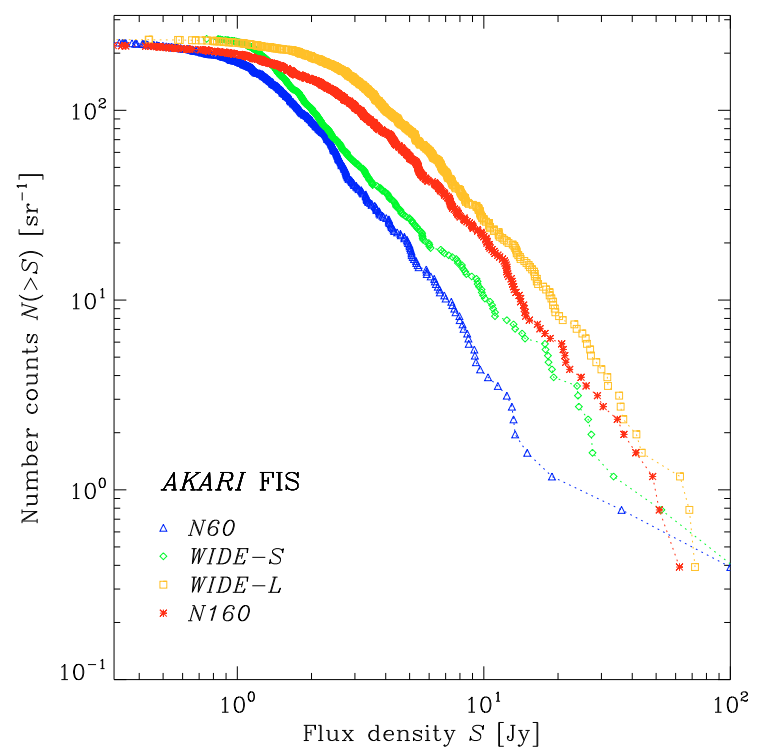

Fig. 2. Number counts of our AKARI multiband sample at the four AKARI FIS bands. Triangles, diamonds, squares, and crosses represent the AKARI N60, WIDE-S, WIDE-L, and N160 galaxy counts, respectively.

$20^{\prime \prime}$ radius. However, again no 2 MASS counterparts were found, and we adopted the standard 2MASS XSC upper limits for these galaxies at $J-, H$-, and $K$ s-bands. The standard 2MASS limiting magnitudes at $J, H$, and $K \mathrm{~s}$ are $14.7,13.9$ and $13.1 \mathrm{mag}$, respectively (Jarrett et al. 2000).

We also matched the AKARI-GALEX sample to the SDSS using the GALEX coordinates for the AKARI-GALEX objects and a search radius of $15^{\prime \prime}$. For the SDSS large resolved galaxies like those we are dealing with here are shredded in multiple detections during the deblending step of the pipeline. We used the closest SDSS match to the AKARI object to obtain the SDSS photometry of the parent object, namely the object detected by the SDSS pipeline before deblending. We inspected all sources to check that the actual flux measured for the parent object is not contaminated by nearby bright stars, artifacts etc.

For most of the sample galaxies, SDSS galaxies were associated. Still, stars superposed on the SDSS galaxy images often hamper accurate photometry. It requires a very careful masking of the SDSS image, and in the current analysis we simply omitted these galaxies. After this selection 607 galaxies remained. We call this sample "the final sample". Almost all galaxies have very secure photometric data at the UV, optical, NIR, MIR, and FIR and the redshift information.

\section{Basic properties of the sample galaxies}

\subsection{Number counts}

Figure 2 presents the cumulative AKARI flux density distribution (number counts) of our final sample. We see that the flux density limit of the sample at $90 \mu \mathrm{m}$ is $\sim 1 \mathrm{Jy}$, shallower than the original limit of $\sim 0.8 \mathrm{Jy}$, because of the additional constraints to have optical-NIR counterparts.

\subsection{Redshift and luminosity distributions}

The redshift distribution of our final sample is shown in Fig. 3. Since the AKARI FIS BSC is rather shallow, most galaxies of the sample are located at low redshifts of $z \lesssim 0.05$. This is quite

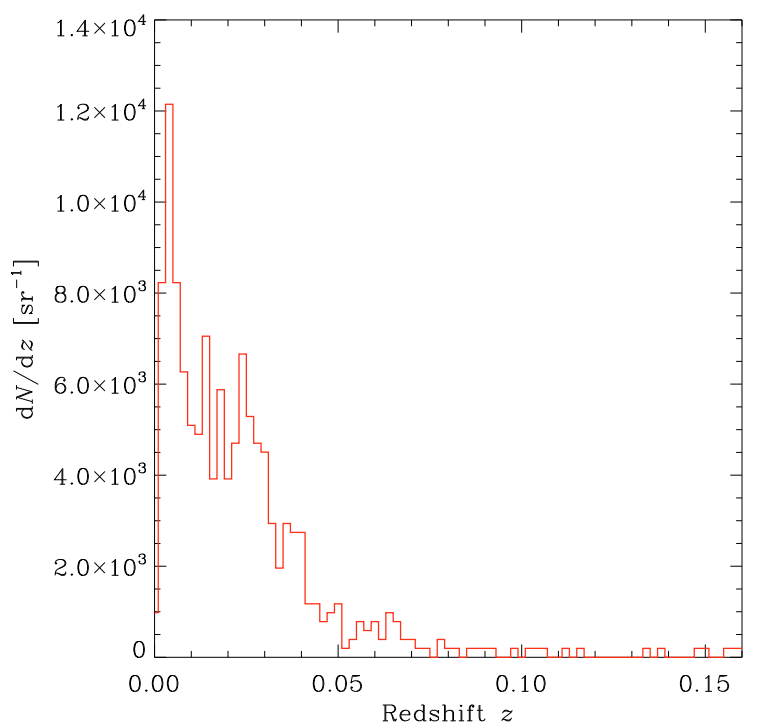

Fig. 3. Redshift distribution of the sample. The distribution is normalized so that we obtain the number of galaxies per unit solid angle if we integrate it over redshifts.

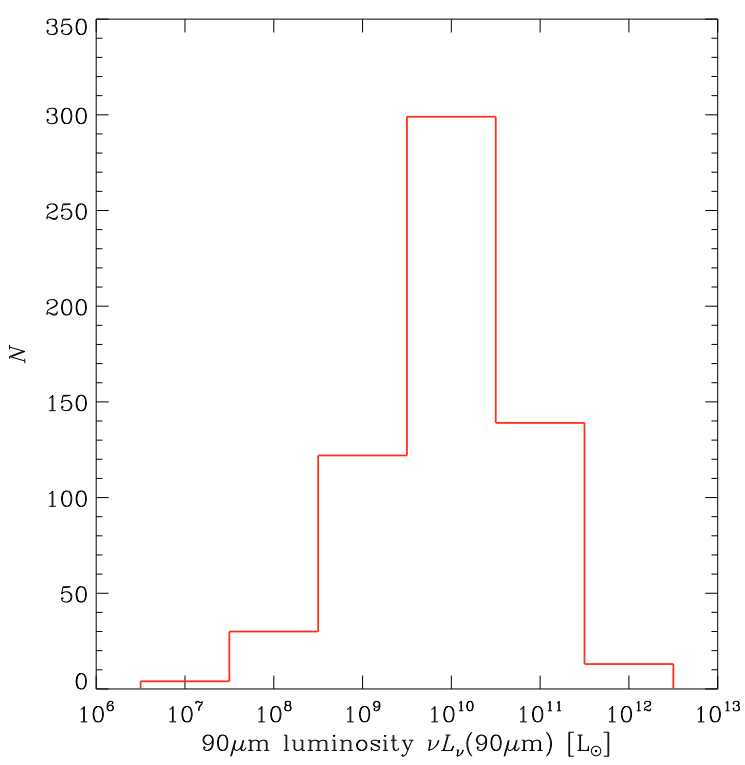

Fig. 4. Luminosity distribution of the sample at $90 \mu \mathrm{m}$.

consistent with predictions of various IR galaxy evolution models (e.g. Takeuchi et al. 2001a,b; Chary \& Elbaz 2001).

Figure 4 presents the $90-\mu \mathrm{m}$ luminosity distribution of the sample. Since this is a raw luminosity distribution of IR galaxies, it decreases toward the faint end. The $90-\mu \mathrm{m}$ luminosity ranges from $10^{6}-10^{12} L_{\odot}$. Only a few galaxies are classified as ultraluminous IR galaxies (ULIRGs). The peak of the luminosity distribution is around $10^{10} L_{\odot}$, which is more luminous than the knee of the $60-\mu \mathrm{m}$ luminosity function (Takeuchi et al. 2003a). Since our selection procedure is multifold and complicated, estimating a reliable luminosity function is not straightforward. We will attempt this task in future works.

We can compare the distribution of galaxies on the redshiftluminosity plane to see the effect of the shape of luminosity function. We show the $z-v L_{v}$ relation in Fig. 5. Some representative wavelengths are shown: AKARI WIDE-S, GALEX FUV, SDSS $r$, and 2MASS Ks. We see that the luminosity at $90 \mu \mathrm{m}$ increases monotonically toward higher redshifts, while the 


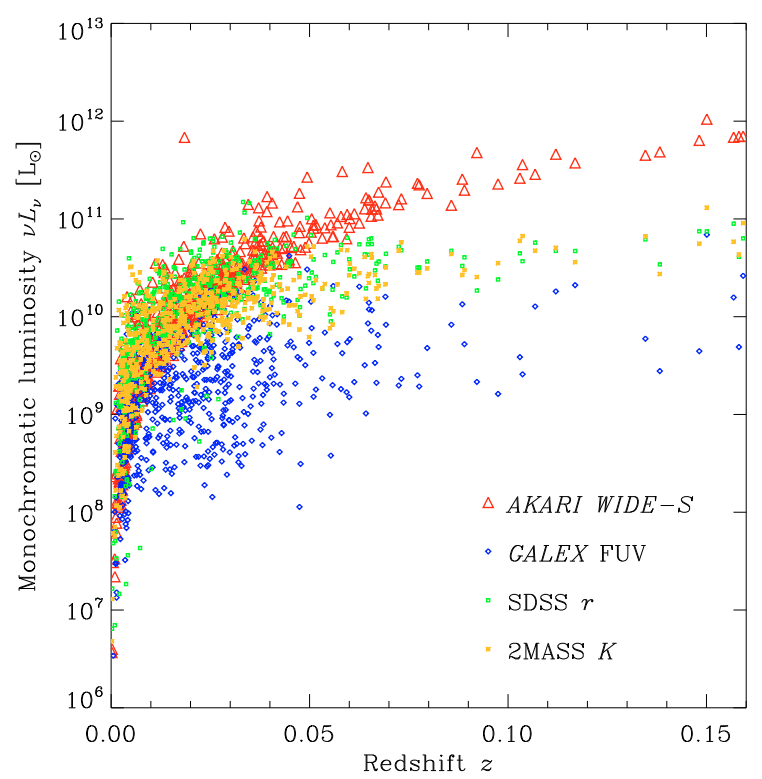

Fig. 5. Luminosity distribution of the sample at several wavelengths. Triangles, diamonds, squares, and crosses represent the galaxies at AKARI WIDE-S, GALEX FUV, SDSS $r$, and 2MASS $K$-band, respectively.

optical and near-IR (NIR) luminosities of the same galaxies saturate at certain values. This is related to the difference in the shape of their luminosity functions (see e.g. Takeuchi et al. 2005a; Iglesias-Páramo et al. 2006); i.e. the luminous end of the function exponentially declines at the optical-NIR and UV, while it shows a power-law decline at the FIR. This is also related to the phenomenon that the more luminous galaxies are more strongly extinguished by dust. We will revisit this issue in a much more direct way in Sect. 4.4.

\subsection{SEDs of the sample}

Since we have the monochromatic luminosities from the FUV to FIR, we construct the spectral energy distributions (SEDs) of our sample. We show all the SEDs of the sample in Fig. 6. Large empty squares represent the AKARI FIS measurements, while the small squares represent all the other data in Fig. 6. We observe a very large variety of SEDs among the sample galaxies.

To see a global trend of the SEDs more clearly, we sorted the sample by their $90-\mu \mathrm{m}$ luminosities and subdivided the sample into six logarithmic bins from $10^{6} L_{\odot}$ to $10^{12} L_{\odot}$ (with a bin width of $\Delta \log L_{90}=1$ ). By taking an average and median, we constructed "average SEDs" as a function of $90-\mu \mathrm{m}$ luminosity, shown in Fig. 7. Because of a large dispersion and asymmetric distribution of the SEDs in each bin, sometimes the average and median SEDs do not agree very well. Even so, the trend is more clearly seen now: low $90-\mu$ m luminosity galaxies have a cooler dust emission and bluer UV-optical continuum, while high $90-\mu$ m luminosity galaxies have a hotter dust emission and redder UV-optical continuum.

The 90- $\mu$ m luminosity dependence of the dust emission temperature is even more clearly seen if we plot a flux density ratio $S_{65} / S_{90}$ as a function of $L_{90}$. This is shown in Fig. 8. Again, the agreement between average and median values is not excellent, we see a clear monotonically increasing trend of the flux density ratio along with $L_{90}$.

The lowest luminosity galaxies have the highest uncertainty mainly because of poor statistics. This problem will be solved

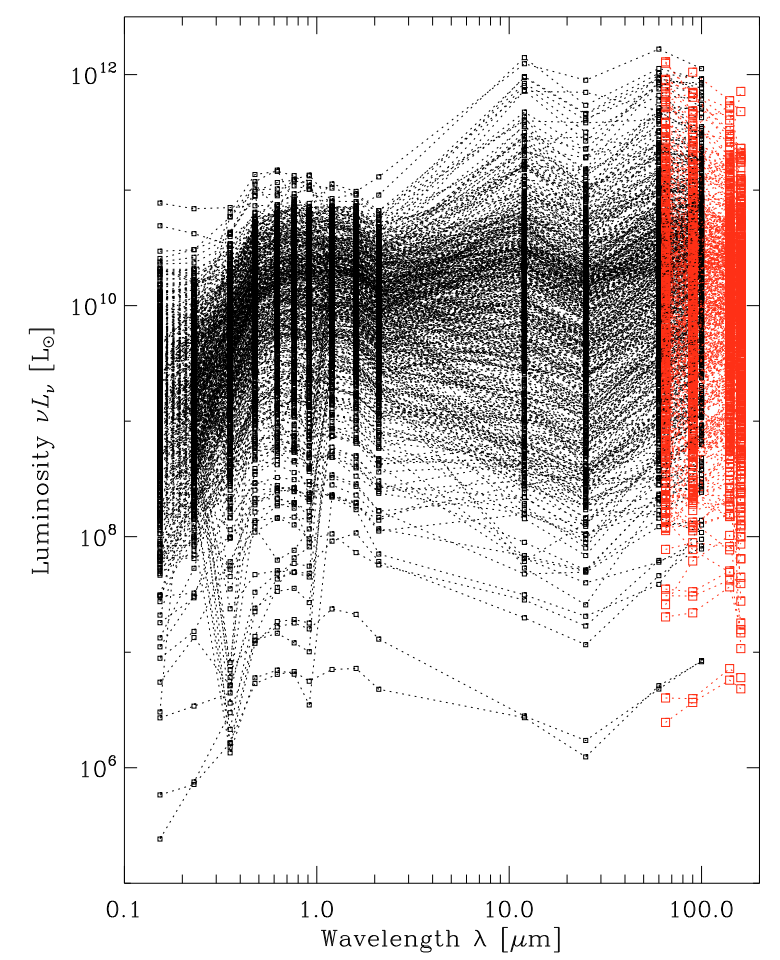

Fig. 6. Spectral energy distributions (SEDs) of the whole sample. Large empty squares represent the AKARI measurements, while the small squares are data taken from GALEX FUV, GALEX NUV, SDSS $u, g$, $r, i, z, 2 \mathrm{MASS} J, H, K \mathrm{~s}$, and IRAS $12,25,60$, and $100 \mu \mathrm{m}$ from left to right. Dotted lines connect data points of each object to guide the eye.

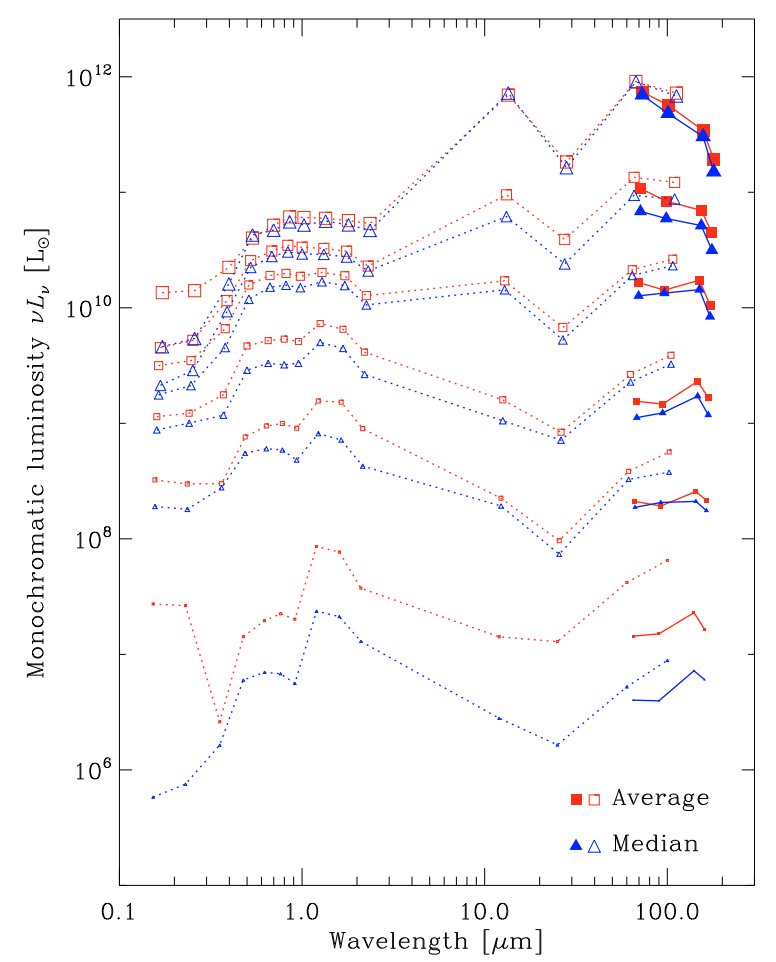

Fig. 7. Averaged SEDs of our sample as a function of $90 \mu \mathrm{m}$ luminosity. Luminosity bins used here are the same as the bins in Fig. 4. The central luminosities of the bins are $10^{7}, 10^{8}, 10^{9}, 10^{10}, 10^{11}$, and $10^{12} L_{\odot}$, respectively, and the obtained average SEDs are represented with different size of the symbols: from the smallest corresponding to $10^{7} L_{\odot}$ to the largest corresponding to $10^{12} L_{\odot}$. Filled and empty squares are averaged SEDs, while filled and empty triangles are median SEDs. 


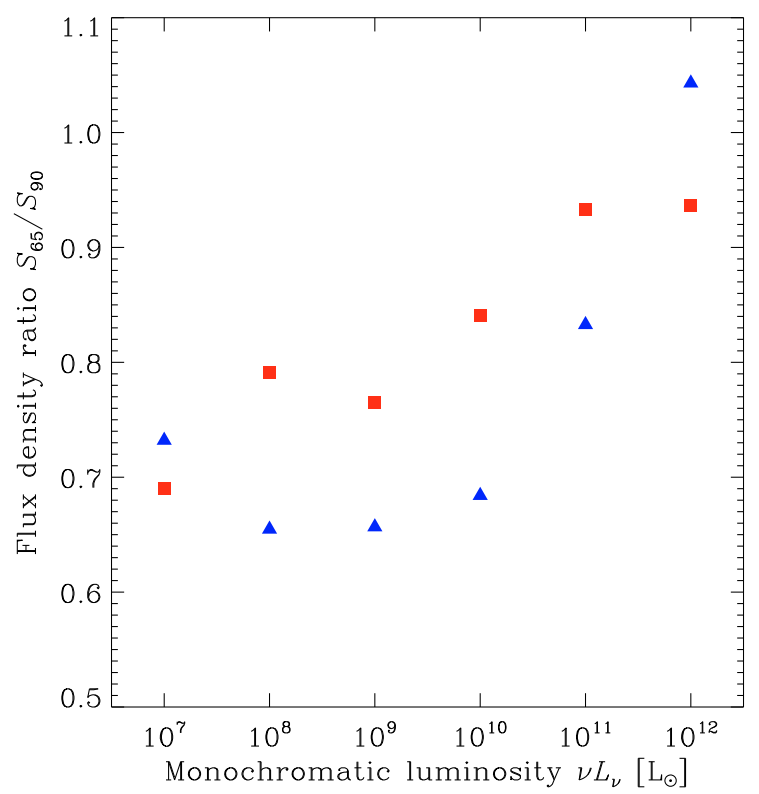

Fig. 8. Dependence of the flux density ratio $S_{65} / S_{90}$ on average $90-\mu \mathrm{m}$ luminosity. Same as Fig. 7, the squares are estimated from averaged SEDs, while triangles are from median SEDs.

by constructing a deeper and more substantial sample, possibly by the next generation AKARI FIS catalog. The most $90-\mu \mathrm{m}$ luminous galaxies seem to have an upturn at the UV. This may be because of an AGN component in these galaxies. We will examine this issue in our future work. From both Figs. 6 and 7 it is clear that AKARI FIS measurements at N60 and WIDE-S do not agree with the IRAS ones. This discrepancy is mostly due to the better angular resolution of the AKARI FIS compared with IRAS. Thus the measured values are far less contaminated by Galactic cirrus emission. Also because of the better angular resolution, the source confusion effect is much smaller than for IRAS. As both cirrus and source confusion effects cause flux boosting for IR galaxy number counts, the IRAS flux densities could be overestimated (Takeuchi \& Ishii 2004). Jeong et al. (2007) also examined the same problem with an early AKARI sample and concluded that the difference between the IRAS and AKARI flux densities is due to the confusion effects. We see a strange bump at $12 \mu \mathrm{m}$ for luminous galaxies. This may not be a physical feature in the SEDs of our sample but may be caused by a poor measurement at this band by IRAS. In the redshift-luminosity diagram at $12 \mu \mathrm{m}$ (see Fig. A.1) we see that many galaxies locate on the limiting luminosity line. This means that even if they are classified as measurements, they are actually the upper limits of the flux density. We plan to study this point in greater depth with the AKARI IRC all-sky survey in the future.

\subsection{The NUV - r distribution}

The NUV $-r$ restframe color is a very efficient tool to separate the galaxies into blue and red populations (Salim et al. 2007; Martin et al. 2007). At high-z, IR selected galaxies observed with Spitzer were found to populate the so-called green valley between the blue cloud and red sequence, first mentioned by Bell et al. (2005) and further identified in the COSMOS fields (Kartaltepe et al. 2009; Vergani et al. 2009).

Yet no systematic study of the IR selected galaxies has been performed in the nearby universe until now. Figure 9 shows

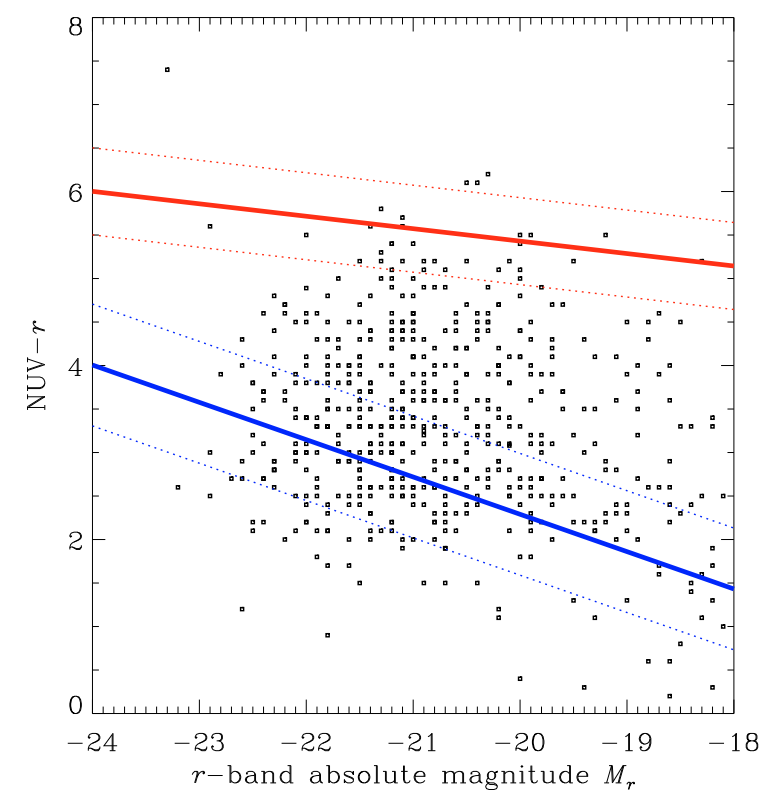

Fig. 9. The absolute $r$-magnitude-NUV $-r$ color distribution. Upper and lower thick solid lines represent the average red sequence and blue cloud taken from Salim et al. (2007), with its envelope indicated by dotted lines.

the distribution of the NUV $-r$ color for our sample galaxies against the absolute magnitude at $r$-band, $M_{r}$. The distribution in the NUV $-r$ is found to be large and monolithic in contrast to the bimodal distribution found in optical surveys. From Fig. 1 of Salim et al. (2007) (based on an SDSS-GALEX selected sample), we approximate the red sequence on this diagram with a linear relation

NUV $-r=-0.143 M_{r}+2.57$,

with \pm 0.5 envelopes, and the the blue cloud with

$\mathrm{NUV}-r=-0.429 M_{r}-6.29$,

with \pm 0.7 . We clearly see that our galaxies lie in the blue cloud and populate the green valley to produce a continuous distribution, and only a few objects are located in the red sequence. Therefore, the color distribution of the FIR-selected galaxies is different than the one obtained from an optical selection, as they preferentially populate the green valley between the red sequence and blue cloud. An interpretation of this behavior in terms of dust attenuation, SF history or AGN activity will be developed in a future paper.

\section{Star formation and dust extinction of the $90 \mu \mathrm{m}$-selected galaxies}

\subsection{Total dust luminosity from the AKARI FIS bands}

To calculate the SF- and attenuation-related physical properties of the galaxies, a TIR luminosity is required. Various estimators of $L_{\mathrm{TIR}}$ were proposed by previous authors (Helou et al. 1988; Dale et al. 2001; Dale \& Helou 2002; Sanders \& Mirabel 1996). Takeuchi et al. (2005b) showed that we can safely estimate the TIR luminosity by a formula proposed by Sanders \& Mirabel (1996)

$$
\begin{aligned}
L_{\mathrm{TIR}} \equiv & 4.93 \times 10^{-22}\left[13.48 L_{v}(12 \mu \mathrm{m})+5.16 L_{v}(24 \mu \mathrm{m})\right. \\
& \left.+2.58 L_{v}(60 \mu \mathrm{m})+L_{v}(100 \mu \mathrm{m})\right]\left[L_{\odot}\right] .
\end{aligned}
$$


T. T. Takeuchi et al.: Star formation and dust extinction of galaxies from AKARI-GALEX
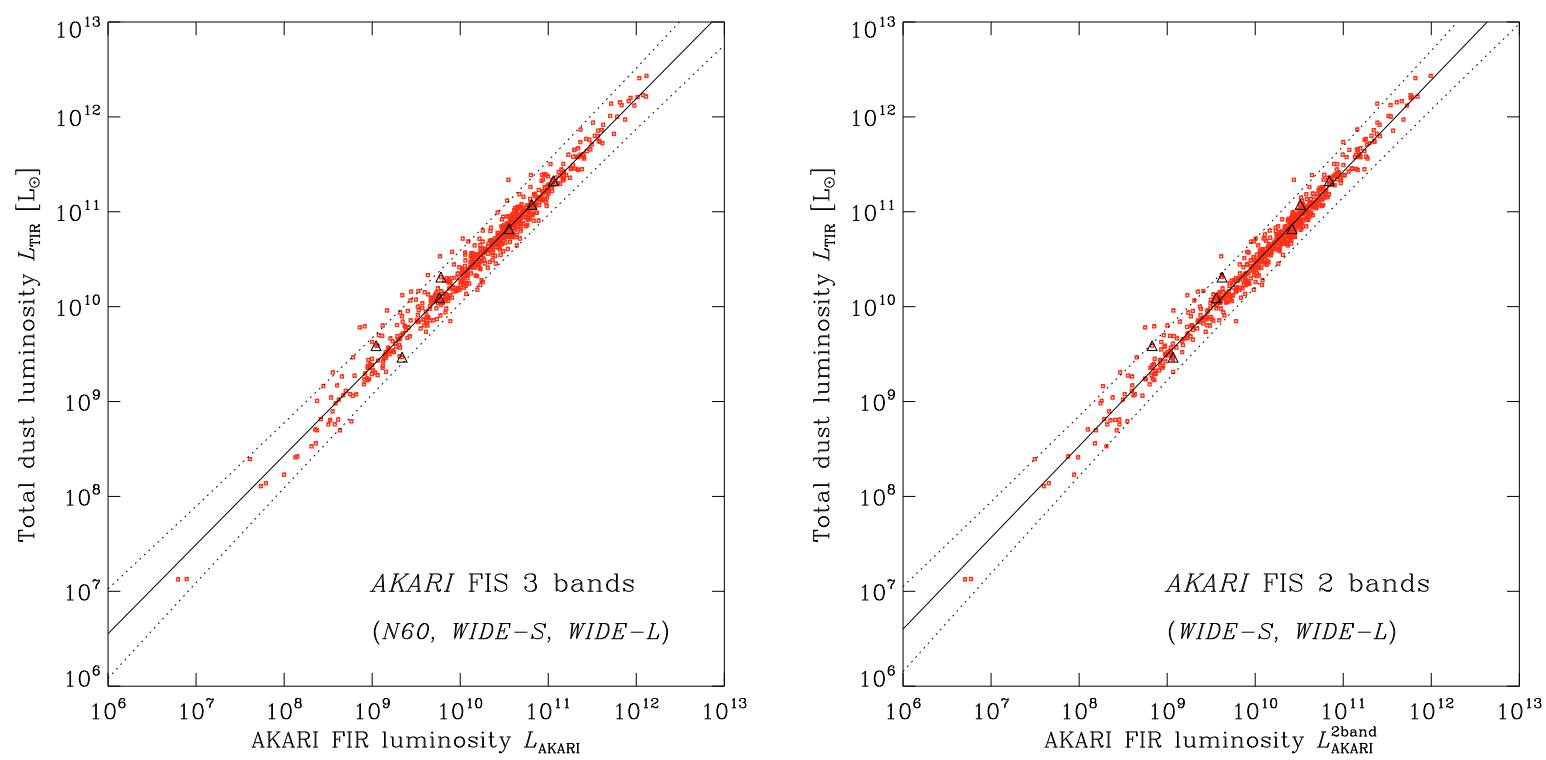

Fig. 10. Relation between the AKARI FIR luminosity and total IR (TIR) luminosity of the sample. The left panel is for the AKARI FIR luminosity defined by Eq. (4), while the right panel is for the AKARI FIR luminosity from two bands, defined by Eq. (8). The black solid lines are the best least-square fits to the data, and the dotted curves represent the $95 \%$ confidence levels of the lines. Triangles are galaxies with an IRAS $100 \mu \mathrm{m}$ quality flag larger than three, i.e., those with an insecure flux density measurement.

Since our sample contains all the IRAS band flux densities, we can use the estimator of Sanders \& Mirabel to examine the AKARI FIR flux. In this subsection we make an attempt to establish a reliable formula to convert a FIR luminosity measured by AKARI to the TIR luminosity.

Since the AKARI FIS has continuous bands from $\sim 50 \mu \mathrm{m}$ to $\sim 160 \mu \mathrm{m}$, we can easily define the FIR flux simply by adding the flux densities multiplied with their bandwidths (in [Hz]). Hirashita et al. (2008) has defined the AKARI FIR luminosity as

$$
\begin{aligned}
L_{\mathrm{AKARI}}= & \Delta v(N 60) L_{v}(65 \mu \mathrm{m})+\Delta v(W I D E-S) L_{v}(90 \mu \mathrm{m}) \\
& +\Delta v(W I D E-L) L_{v}(140 \mu \mathrm{m}),
\end{aligned}
$$

where

$$
\begin{aligned}
& \Delta v(N 60)=1.58 \times 10^{12}[\mathrm{~Hz}] \\
& \Delta v(W I D E-S)=1.47 \times 10^{12}[\mathrm{~Hz}] \\
& \Delta v(W I D E-L)=0.831 \times 10^{12}[\mathrm{~Hz}] .
\end{aligned}
$$

However, since the sensitivity of AKARI N60 is not as good as other two wide bands, it will be useful if we can use the FIR luminosity defined only by WIDE-S and WIDE-L, because we obtain more substantial number of galaxies that way. We define the $L_{\text {AKARI }}^{2 \text { band }}$ by omitting the term of $N 60$ as

$$
\begin{aligned}
L_{\mathrm{AKARI}}^{2 \mathrm{band}}= & \Delta v(W I D E-S) L_{v}(90 \mu \mathrm{m}) \\
& +\Delta v(W I D E-L) L_{v}(140 \mu \mathrm{m}) .
\end{aligned}
$$

Figure 10 presents the correlations between $L_{\mathrm{AKARI}}$ and $L_{\mathrm{TIR}}$. The left panel shows the correlation between $L_{\text {AKARI }}$ of Hirashita et al. (2008) and $L_{\mathrm{TIR}}$, while the right panel shows the one between the $L_{\mathrm{AKARI}}^{2 \mathrm{band}}$ and $L_{\mathrm{TIR}}$. The fitting results are

$\log L_{\mathrm{TIR}}=0.940 \log L_{\mathrm{AKARI}}+0.914$,

$r=0.987$,

and

$\log L_{\mathrm{TIR}}=0.964 \log L_{\mathrm{AKARI}}^{2 \mathrm{band}}+0.814$,

$r=0.989$, where $r$ is the correlation coefficient. The solid lines in Fig. 10 depict these equations. The envelopes delineated by dotted lines in Fig. 10 represent the $95 \%$ confidence intervals.

In some cases the quality of the IRAS $100 \mu \mathrm{m}$ flux density measurement is poor; we examine the impact of these objects in Fig. 10. Triangles represent galaxies with an IRAS $100 \mu \mathrm{m}$ quality flag larger than three, i.e. those for which the measurement was difficult and not secure. However, even so they clearly lie well within the distribution of the whole sample and do not have a significant impact on the statistical analysis.

Since these plots present luminosity-luminosity correlations, the very tight correlations are not extremely surprising. Still, the uncertainties of these equations are within a factor of $\sim 2-3$, which means that the estimation works very well even if we do not have MIR measurements. We also stress that the correlation is even better if we only use the AKARI wide bands.

Below we use the total IR luminosity $L_{\mathrm{TIR}}$ obtained from the AKARI N60, WIDE-S, and WIDE-L (Eq. (9)) for the analysis, that is, when we use $L_{\mathrm{TIR}}$, the AKARI-based total IR luminosity is meant.

\subsection{Luminosity from current star formation}

Now we can compare the FUV $\left(L_{\mathrm{FUV}}\right)$ and TIR $\left(L_{\mathrm{TIR}}\right)$ luminosities for our final sample. This is shown in Fig. 11. It is striking that the luminosity is dominated by $L_{\mathrm{TIR}}$ for the vast majority of our sample, even though we take into account that they are FIR-selected. It is also worth mentioning that the luminosity at the FUV does not exceed $10^{11} L_{\odot}$. In contrast, $L_{\mathrm{TIR}}$ can be much higher.

By combining $L_{\mathrm{FUV}}$ and $L_{\mathrm{TIR}}$, we can define the luminosity contribution from newly formed stars, $L_{\mathrm{SF}}$

$L_{\mathrm{SF}} \equiv L_{\mathrm{FUV}}+(1-\eta) L_{\mathrm{TIR}}$

where $\eta$ is the fraction of the dust emission due to the heating of grains by old stars which is not directly related to the recent SFR. We adopt a value of $30 \%$ for this fraction (Hirashita et al. 2003). The contribution of $L_{\mathrm{FUV}}$ and $L_{\mathrm{TIR}}$ is shown in 


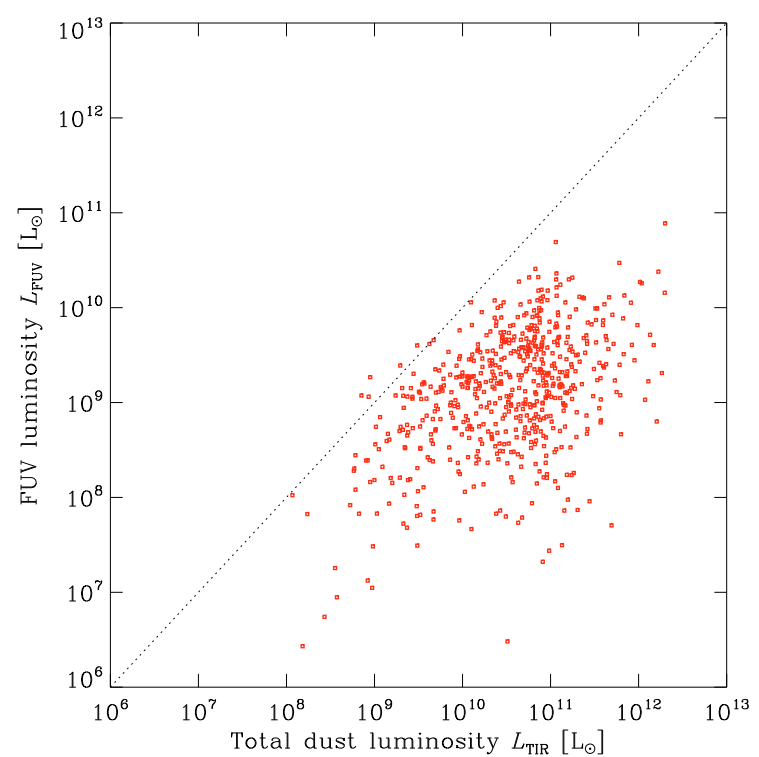

Fig. 11. Comparison between TIR and FUV luminosities. The diagonal dotted line represents the case if $L_{\mathrm{TIR}}$ equals $L_{\mathrm{FUV}}$.

Fig. 12. Naturally the contribution of $(1-\eta) L_{\mathrm{TIR}}$ dominates $L_{\mathrm{SF}}$. In contrast, the contribution of $L_{\mathrm{FUV}}$ has a very large scatter, and the correlation is very poor. Hence it is not surprising that it is very difficult to estimate the total energy emitted by newly formed stars only from FUV information, even in an average sense. The UV contribution becomes significant at lower luminosities of $L_{\mathrm{SF}}<10^{10} L_{\odot}$ as seen in the left panel of Fig. 12.

\subsection{Star formation rate of galaxies in our sample}

Here we interpret the data in terms of the SFR. Assuming a constant SFR over $10^{8} \mathrm{yr}$ and the Salpeter initial mass function (IMF) (Salpeter 1955, mass range: 0.1-100 $M_{\odot}$ ), we have the relation between the $\mathrm{SFR}$ and $L_{\mathrm{FUV}}$

$\log \mathrm{SFR}_{\mathrm{FUV}}=\log L_{\mathrm{FUV}}-9.51$.

To transform the dust emission to the SFR for the IR, we assume that all stellar light is absorbed by dust. Then we obtain the following formula under the same assumption for both the SFR history and the IMF as those of the FUV

$$
\log \mathrm{SFR}_{\text {dust }}=\log L_{\mathrm{TIR}}-9.75+\log (1-\eta) \text {. }
$$

Here $\eta$ is again the fraction of the dust emission by old stars. Thus the total SFR is simply

$\mathrm{SFR}=\mathrm{SFR}_{\mathrm{FUV}}+\mathrm{SFR}_{\text {dust }}$.

The obtained SFR is shown as a function of the fraction of the contribution of the SFR $\mathrm{FUV}$ in Fig. 13. Reflecting the large scatter of $L_{\mathrm{FUV}} / L_{\mathrm{SF}}$, the scatter of the $\mathrm{SFR}_{\mathrm{FUV}} / \mathrm{SFR}$ is very large at $\mathrm{SFR}<20 M_{\odot} \mathrm{yr}^{-1}$. However, there is a sudden drop of the $\mathrm{SFR}_{\mathrm{UV}}$ contribution at SFR $>20 M_{\odot} \mathrm{yr}^{-1}$. The vertical dotted line shows this "threshold" SFR in Fig. 13.

\subsection{Dust attenuation}

\subsubsection{Dust attenuation of the sample}

Galaxies selected in the IR are expected to have quite a high dust attenuation. Here we examine the extinction properties of galaxies in the sample. For this study a good observational indicator of dust attenuation is required. The $L_{\mathrm{TIR}} / L_{\mathrm{FUV}}$ ratio is widely recognized to be a robust measure of dust attenuation. This ratio was found to increase with the star formation luminosity in a similar way from $z=0$ to $z=0.7$ (Martin et al. 2005; Buat et al. 2007b; Zheng et al. 2006). Dust attenuation can be derived with the formula of Buat et al. (2005)

$$
\begin{aligned}
A_{\mathrm{FUV}}= & -0.0333\left(\log \frac{L_{\mathrm{TIR}}}{L_{\mathrm{FUV}}}\right)^{3} \\
& +0.3522\left(\log \frac{L_{\mathrm{TIR}}}{L_{\mathrm{FUV}}}\right)^{2} \\
& +1.1960\left(\log \frac{L_{\mathrm{TIR}}}{L_{\mathrm{FUV}}}\right)+0.4967[\mathrm{mag}]
\end{aligned}
$$

Figure 14 presents the variation of $L_{\mathrm{TIR}} / L_{\mathrm{FUV}}$ as a function of the star formation luminosity. A clear increase of $L_{\mathrm{TIR}} / L_{\mathrm{FUV}}$ with $L_{\mathrm{SF}}$ is seen. The solid line is the mean trend of local galaxies found in our IRAS study (Buat et al. 2007a)

$\log \left(\frac{L_{\mathrm{TIR}}}{L_{\mathrm{FUV}}}\right)=0.64 \log L_{\mathrm{SF}}-5.5$.

This line was estimated by a weighting of $L_{\mathrm{TIR}} / L_{\mathrm{FUV}}$ with $1 / V_{\max }$ to eliminate the flux selection effect, i.e. the effect that more luminous galaxies can be more easily detected. In contrast, we plot raw values of $L_{\mathrm{TIR}} / L_{\mathrm{FUV}}$. However, though the steep rise of the distribution of $L_{\mathrm{TIR}} / L_{\mathrm{FUV}}$ with $L_{\mathrm{SF}}$ is partially because of this selection effect, the trend is still well consistent with our IRAS study (Buat et al. 2007a), and the conclusion would remain valid.

\subsubsection{Dust attenuation versus the slope of the UV continuum}

The slope of the UV continuum is commonly used as a proxy to estimate dust attenuation and $L_{\mathrm{TIR}} / L_{\mathrm{FUV}}$ when IR data are not available or considered as unreliable (Daddi et al. 2007; Reddy et al. 2008; Reddy \& Steidel 2009). This method is based on a calibration performed on local starburst galaxies (Meurer et al. 1995, 1999). GALEX observations brought a large amount of data in this field since the slope of the UV continuum can be safely deduced from the FUV - NUV color. Studies based on the GALEX data have shown that the local starburst calibration is not valid for the bulk of local star forming galaxies (e.g. Dale et al. 2005; Cortese et al. 2006; Boissier et al. 2007; Gil de Paz et al. 2007; Johnson et al. 2007): i.e. these galaxies exhibit lower dust attenuation $\left(L_{\mathrm{TIR}} / L_{\mathrm{FUV}}\right)$ than expected from their FUV - NUV color. IR selected galaxies also depart from the starburst law but in the opposite way: they are found to exhibit a $L_{\mathrm{TIR}} / L_{\mathrm{FUV}}$ ratio much higher than expected (Buat et al. 2005; Goldader et al. 2002). Here we re-investigate this issue making use of a much larger sample than the one used in Buat et al. (2005).

Figure 15 shows the FUV - NUV color against $L_{\mathrm{TIR}} / L_{\mathrm{FUV}}$ (often called the IR-excess (IRX) $-\beta$ relation) for our sample galaxies together with the local starburst relation. The local relation was taken from Meurer et al. (1999) and converted into the relation between the FUV - NUV color and $L_{\mathrm{TIR}} / L_{\mathrm{FUV}}$ by Eqs. (1) and (2) of Kong et al. (2004)

$\frac{L_{\mathrm{TIR}}}{L_{\mathrm{FUV}}}=10^{[1.92(\mathrm{FUV}-\mathrm{NUV})+0.4]}-0.95$.

Most of the galaxies lie below the starburst relation by a factor of five to ten, but some are located above this relation. A significant 
T. T. Takeuchi et al.: Star formation and dust extinction of galaxies from AKARI-GALEX
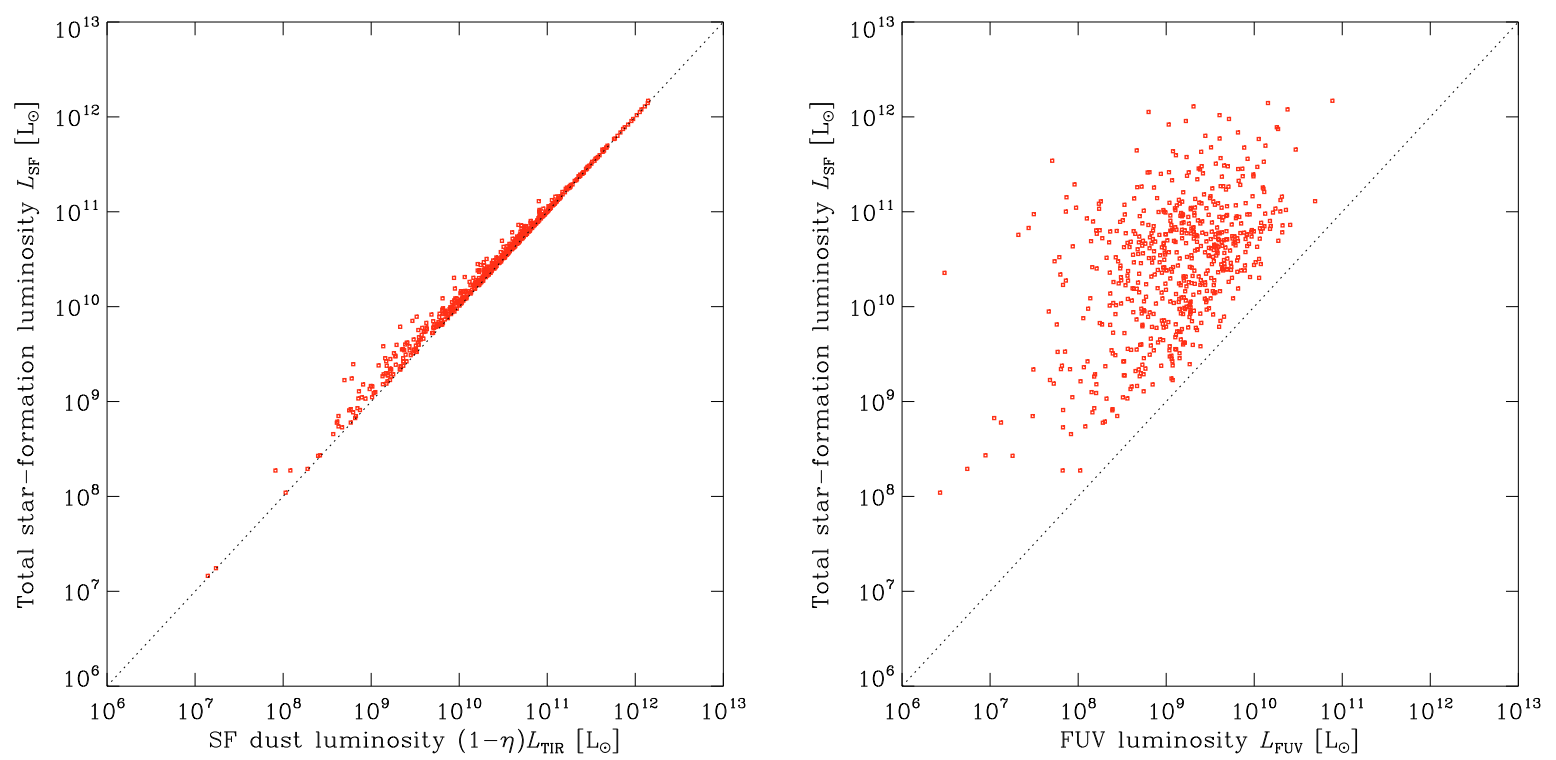

Fig. 12. Contribution of $L_{\mathrm{TIR}}$ and $L_{\mathrm{FUV}}$ to the total star formation (SF) luminosity $L_{\mathrm{SF}}$, which is the luminosity produced by newly forming stars defined by Eq. (13). In the left panel, $\eta$ is the fraction of the IR emission produced by dust heated by old stars which is not related to the current SF (see main text).

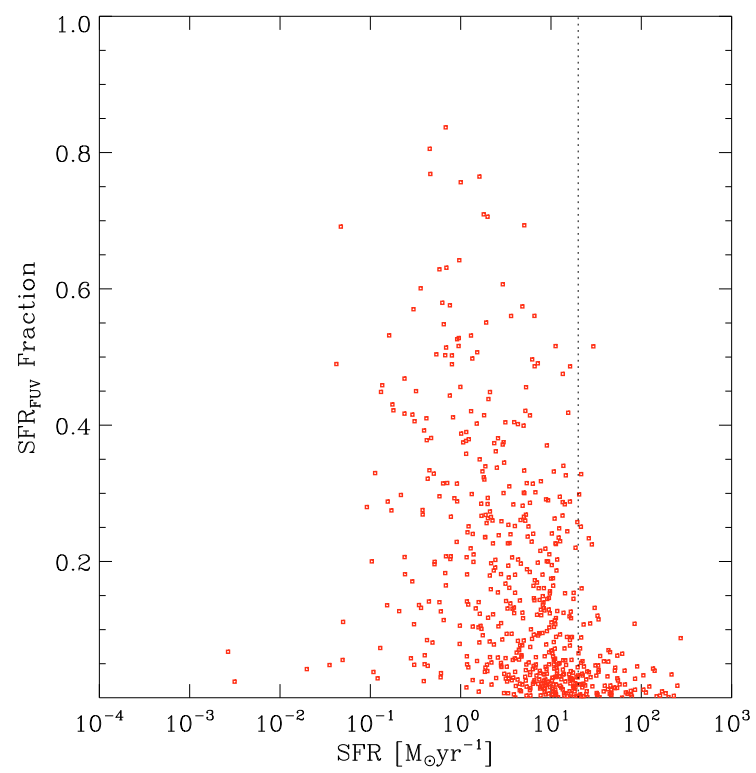

Fig. 13. The contribution of the FUV-estimated (or "directly visible") SFR, SFR $_{U V}$, to the total SFR as a function of the total SFR. The vertical dotted line represents the effective boundary above which almost all energy produced by newly forming stars is emitted at IR.

fraction of galaxies above the local starburst line are luminous IR galaxies (LIRGs and ULIRGs). Especially all the ULIRGs have larger $L_{\mathrm{TIR}} / L_{\mathrm{FUV}}$ ratios than expected from the relation. This general trend is quite consistent with Buat et al. (2005). It may be worth mentioning that LIRGs are consistent with the local starburst line. These trends are more clearly represented by functional fits in the right panel of Fig. 15. We used a functional form of Eq. (19) for "normal" galaxies with $L_{60}<10^{11} L_{\odot}$,

$\frac{L_{\mathrm{TIR}}}{L_{\mathrm{FUV}}}=10^{[a(\mathrm{FUV}-\mathrm{NUV})+b]}-c$.

with the three parameters, $a, b$ and $c$ free. The fitted relation is well below the local starburst relation originally claimed by Meurer et al. (1999). For more luminous galaxies Eq. (20) does

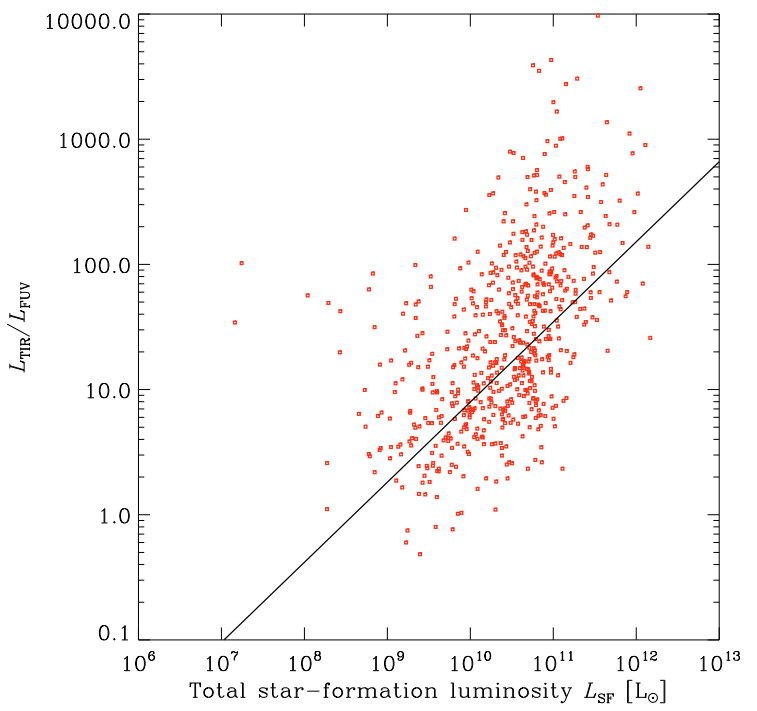

Fig. 14. The distribution of the $L_{\mathrm{TIR}}-L_{\mathrm{FUV}}$ ratio as a function of the star formation luminosity $L_{\mathrm{SF}}$. The solid line is the relation found by the IRAS-GALEX analysis (Buat et al. 2007a), represented by Eq. (18).

not give a reasonable fit, hence we adopted a simple linear fit. Even if LIRGs are roughly consistent with the local starburst relation, the fitted line is much flatter than that because of the existence of galaxies well above the local starburst line with blue UV color. It is difficult to come to a conclusion for the ULIRGs because of the poor statistics, but the fitted linear relation seems to be located above the local starburst relation.

We have seen that galaxies with relatively quiescent galaxies $\left(L_{60}<10^{11} L_{\odot}\right)$ in our sample locate well below the relation of Meurer et al. (1999). A similar trend was reported for optically selected galaxies (e.g. Boissier et al. 2007; Cortese et al. 2006). Boissier et al. (2007) investigated this relation for normal galaxies selected at optical wavelength with the same functional form as this study (Eq. (20)). They found a much flatter relation than Meurer's original relation, which is presented in the right panel of Fig. 15 with the dot-dot-dot-dashed line. Cortese et al. (2006) 

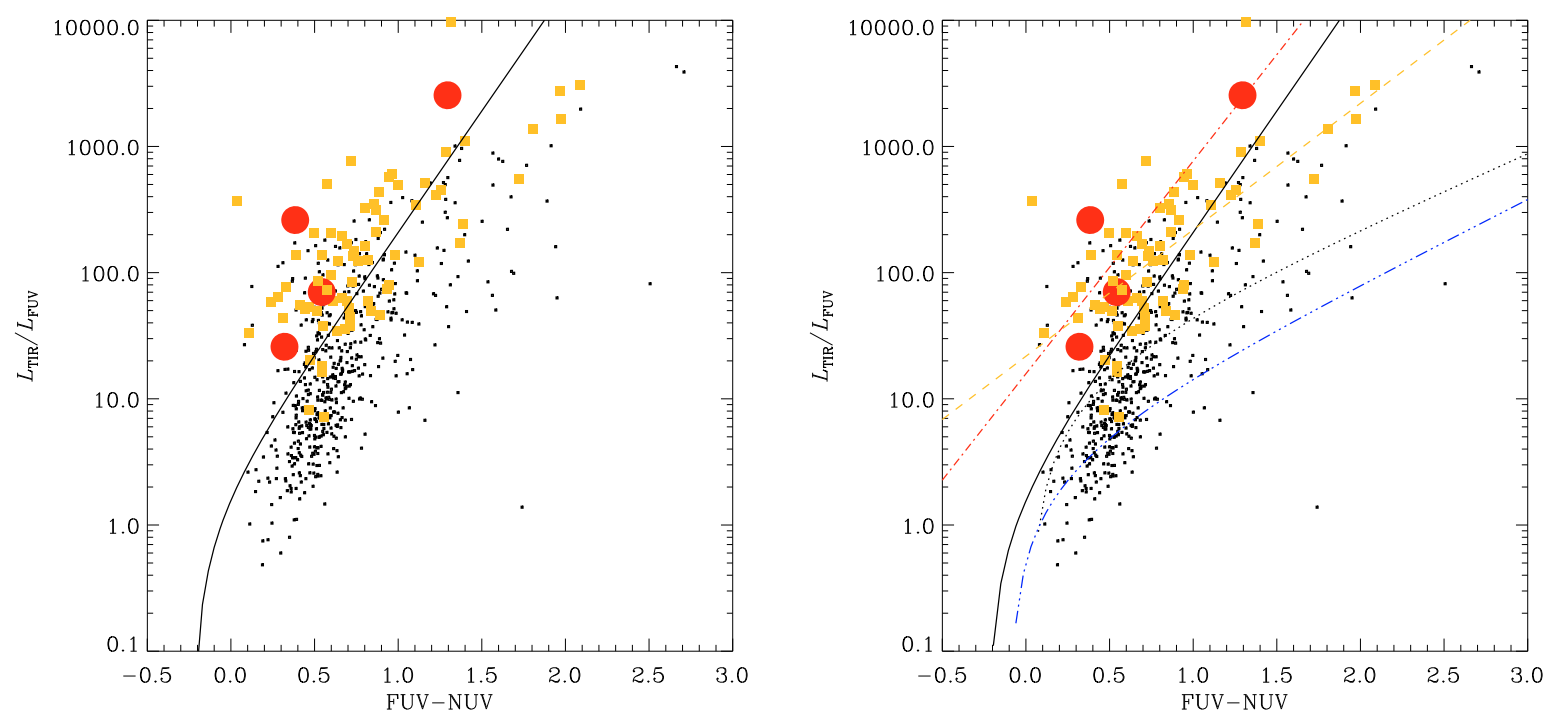

Fig. 15. The distribution of $L_{\mathrm{TIR}}-L_{\mathrm{FUV}}$ luminosity ratio as a function of the UV color FUV - NUV. Dots: galaxies with $60 \mu \mathrm{m}$ luminosity $L_{60}<10^{11} L_{\odot}$, filled squares: galaxies with $10^{11} \leq L_{60}<10^{12} L_{\odot}$ (IR luminous galaxies: LIRGs), and filled circles: galaxies with $10^{11} \leq L_{60}<$ $10^{12} L_{\odot}$ (ultraluminous IR luminous galaxies: ULIRGs). Solid lines in each panel represent the relation for local starbursts proposed by Meurer et al. (1999), which was converted into the relation between the FUV - NUV color (Eq. (19)). The left panel shows the raw distribution of the $L_{\mathrm{TIR}}-L_{\mathrm{FUV}}$ luminosity ratio, while in the right panel the functional fits are overplotted on the data. For galaxies with a $L_{60}<10^{11} L_{\odot}$, Eq. (19) is used, while for more luminous galaxies simple linear relations are adopted because of the poor fit of the function. Dotted line: a fit to galaxies with $L_{60}<10^{11} L_{\odot}$ by Eq. (20); dot-dot-dot-dashed line: a fit to a sample of normal galaxies by Eq. (20) presented by Boissier et al. (2007); dashed line: a linear fit to the LIRGs; dot-dashed line: a linear fit to the ULIRGs.

reported a similar result. What is newly clarified in this study is that the same trend is seen for the purely FIR-selected sample of SF galaxies. In addition, Boissier et al.'s relation does not represent the relation for our quiescent galaxy sample. This is obviously the case because our sample includes galaxies strongly reddened by dust which can never be picked up by UV-selection.

\subsubsection{Attenuation and stellar mass-related quantities}

By using optical-NIR flux densities we can estimate the stellar mass of the sample galaxies. Here we discuss the dust attenuation properties with respect to the stellar mass. There are various methods to estimate stellar mass. In this work, we used an SDSS-based method proposed by Yang et al. (2007); these authors assume the Kroupa IMF (Kroupa 2001), while we used the Salpeter IMF (Salpeter 1955) to estimate the SFR of galaxies. For consistency, we convert their stellar mass estimates with the conversion factor given by Bell et al. (2003). As for the accuracy of the stellar mass estimates, the relation has been calibrated from the SDSS+2MASS data, and the error should be around $20 \%$. We also estimated the stellar mass from $K$ s-band using the relations derived by Bell et al. (2003), and the trends did not change significantly. Other explorations for the stellar mass estimation will be discussed elsewhere.

Figure 16 shows the relation between stellar mass $M_{*}$ and dust attenuation in terms of $L_{\mathrm{TIR}} / L_{\mathrm{FUV}}$. At a first glance the dependence of dust attenuation on the stellar mass is very strong. Though we should be again cautious of the selection effect on this plot, the strong dependence might be partially physical. This implies that larger galaxies are more extinguished. Hence dust attenuation is closely related to the physical size of galaxies.

The presence of low mass and high $L_{\mathrm{TIR}} / L_{\mathrm{FUV}}$ galaxies $\sim 10-100$ at $M_{*} \simeq 10^{7}-10^{9} M_{\odot}$ is worth mentioning here. Iglesias-Páramo et al. (2006) made essentially the same analysis by GALEX and IRAS. In their studies such galaxies did not exist in the IR-selected samples (their Fig. 12). If the flux

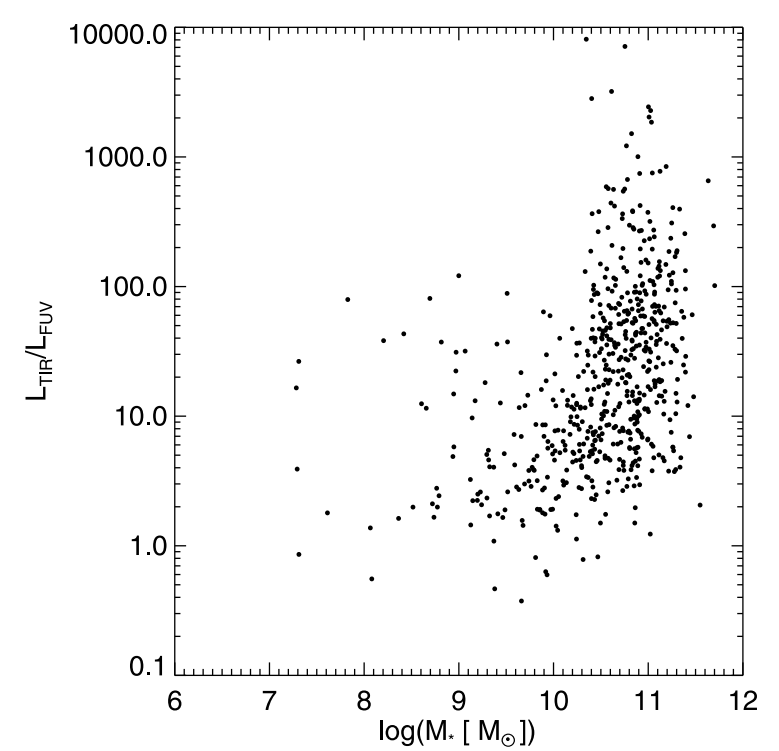

Fig. 16. Relation between stellar mass $M_{*}$ and dust attenuation indicator $L_{\mathrm{TIR}} / L_{\mathrm{FUV}}$.

measurement is secure, these galaxies deserve a close inspection to examine their nature.

Now we show the relation between the SFR per unit stellar mass, $\mathrm{SFR} / M_{*}$, which is often referred to as the specific SFR (SSFR), and the dust attenuation $L_{\mathrm{TIR}} / L_{\mathrm{FUV}}$ in Fig. 17 . It is indeed striking that there is obviously no correlation between these quantities.

A first conclusion would be that there is no correlation between a global attenuation and the SSFR. Generally, many authors showed that the dust attenuation increases with the SFR (see e.g. Fig. 7 of Buat et al. 2007a). But if there is no link with the SSFR it implies that the dust attenuation is linked to the total amount of SF scaled with galaxy size, because the SFR was 


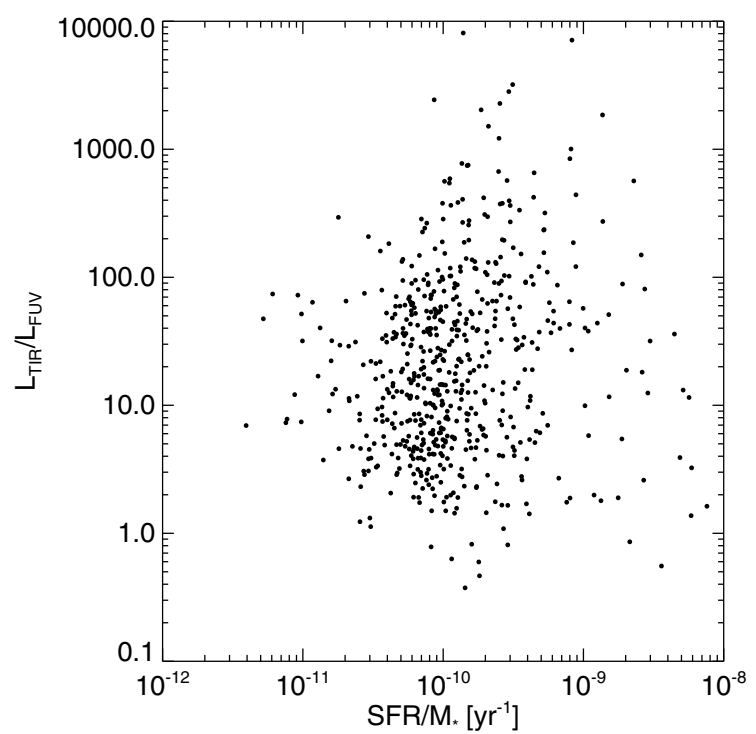

Fig. 17. Relation between SFR per unit stellar mass, SFR/ $M_{*}$ (specific SFR: SSFR), and dust attenuation $L_{\mathrm{TIR}} / L_{\mathrm{FUV}}$.

positively correlated to $M_{*}$ seen above. It may be interpreted as follows: the attenuation is not related to the current-to-past SFR ratio, or roughly, the SF history. This could probably be explained by the short lifetime of dust grains in the ISM.

We should mention though that Iglesias-Páramo et al. (2006) showed that even for the FIR-selected galaxies there is some correlation between these two quantities (see their Fig. 10b). This issue requires further examination.

\section{Conclusion}

In order to explore the SEDs, star formation, and dust extinction properties of galaxies in the Local Universe, we constructed a multiband galaxy sample based on the AKARI FIS all-sky survey and the GALEX all-sky imaging survey (AIS). We started from the AKARI all-sky survey bright source catalogue $\beta-1.1$ and selected galaxies by matching the AKARI sources with those of the IRAS PSC $z$. Then we measured the total GALEX FUV and NUV flux densities by a specially developed photometry software. In a next step we matched this sample to the SDSS and 2MASS galaxies to obtain the basic sample. The basic sample consists of 776 galaxies. After removing objects with photometry contaminated by foregound sources (mainly in the SDSS), we defined the "secure sample" which contains 607 galaxies. Based on this galaxy sample, we explored various properties of galaxies related to star formation and dust extinction.

Summary and conclusions of this study are as follows:

1. The sample galaxies have redshifts $\lesssim 0.15$, and their 90 - $\mu \mathrm{m}$ luminosities range from $10^{6}$ to $10^{12} L_{\odot}$, with a peak at $10^{10} L_{\odot}$.

2. The SEDs display a very large variety, especially more than four orders of magnitude at M-FIR, but if we sort the sample by $90 \mu \mathrm{m}$, their average SED has a coherent trend: the more luminous at $90 \mu \mathrm{m}$, the redder the global SED becomes.

3. The $M_{r}-\mathrm{NUV}-r$ color-magnitude relation of our sample does not show a bimodality which is almost always expected in optically selected galaxy samples. The distribution is unimodal, centered on the green valley between blue cloud and red sequence seen in optical surveys.
4. We have established formulae to convert FIR luminosity from AKARI bands to the TIR luminosity $L_{\mathrm{TIR}}$.

5. The luminosity related to star formation activity $\left(L_{\mathrm{SF}}\right)$ is dominated by the contribution of $L_{\mathrm{TIR}}$ even if we take into account the FIR emission from dust heated by old stars.

6. With these formulae, we calculated the star formation directly visible with FUV and hidden by dust. At high star formation rate (SFR) $\left(>20 M_{\odot} \mathrm{yr}^{-1}\right)$, the fraction of directly visible $\mathrm{SFR}, \mathrm{SFR}_{\mathrm{FUV}}$, decreases.

7. We estimated the ratio, $L_{\mathrm{TIR}} / L_{\mathrm{FUV}}$, which is a direct measure of the FUV attenuation $A_{\mathrm{FUV}}$. The distribution of $L_{\mathrm{TIR}} / L_{\mathrm{FUV}}$ is consistent with a previous result based on GALEX and IRAS (Buat et al. 2007a).

8. We also examined the $L_{\mathrm{TIR}} / L_{\mathrm{FUV}}-\mathrm{UV}$ slope (FUV-NUV) relation. The majority of the sample has $L_{\mathrm{TIR}} / L_{\mathrm{FUV}}$ ratios 5 to 10 times lower than expected from the local starburst relation (Kong et al. 2004), while some LIRGs and all the ULIRGs of this sample have higher $L_{\mathrm{TIR}} / L_{\mathrm{FUV}}$ ratios. This trend was already reported from a previous GALEX-IRAS study (Buat et al. 2005) obtained by a much smaller sample, and we have confirmed their conclusion.

9. By making use of stellar mass information derived from SDSS flux densities in this work, we have examined the dust attenuation properties in terms of stellar mass. We found that the attenuation indicator $L_{\mathrm{TIR}} / L_{\mathrm{FUV}}$ is correlated to stellar mass of galaxies, $M_{*}$, but there is no correlation with SSFR, SFR $/ M_{*}$. This may mean that $L_{\mathrm{TIR}} / L_{\mathrm{FUV}}$ is not linked to the SF history, but simply scales with the size of galaxies. However, this is at odds with previous result of Iglesias-Páramo et al. (2006).

This sample will serve as an important reference sample at $z=0$ for various further analysis or ongoing/future observational projects, like Herschel: for instance, this can be used to construct a set of SEDs for discussing higher- $z$ observational strategy, or as a baseline test sample to investigate a method of extracting galaxies only from FIR flux information (e.g. Pollo et al. 2010).

However, since our first sample is not complete in many senses, further analysis will be desired. We plan to construct a larger multiwavelength sample from the next release of AKARI FIS all-sky survey in the near future.

Acknowledgements. We deeply thank the anonymous referee for her/his careful reading of the original manuscript, useful suggestions and comments which improved the clarity of the paper. This work is based on observations with AKARI, a JAXA project with the participation of ESA. T.T.T. has been supported by Program for Improvement of Research Environment for Young Researchers from Special Coordination Funds for Promoting Science and Technology, and the Grant-in-Aid for the Scientific Research Fund (20740105) commissioned by the Ministry of Education, Culture, Sports, Science and Technology (MEXT) of Japan. V.B. and D.B. have been supported by the Centre National des Etudes Spatiales (CNES) and the Programme National Galaxies (PNG). We thank Agnieszka Pollo, Mai Fujiwara, Akira Ikeyama, Ryosuke Asano, Akio K. Inoue, Hiroshi Shibai, Yasuo Doi, Hideaki Fujiwara, Mitsunobu Kawada, Hidehiro Kaneda, Hiroyuki Hirashita, and Takako T. Ishii for fruitful discussions and comments. T.T.T., F.T.Y., and K.L.M. are partially supported from the Grand-in-Aid for the Global COE Program "Quest for Fundamental Principles in the Universe: from Particles to the Solar System and the Cosmos" from the MEXT.

\section{Appendix A: Luminosity vs. redshift diagrams of the sample}

In Appendix, we show the redshift-luminosity relations for all the bands we have used in the main text (Fig. A.1). These plots help to examine the effect of the selection functions of our sample at each band. 
A\&A 514, A4 (2010)
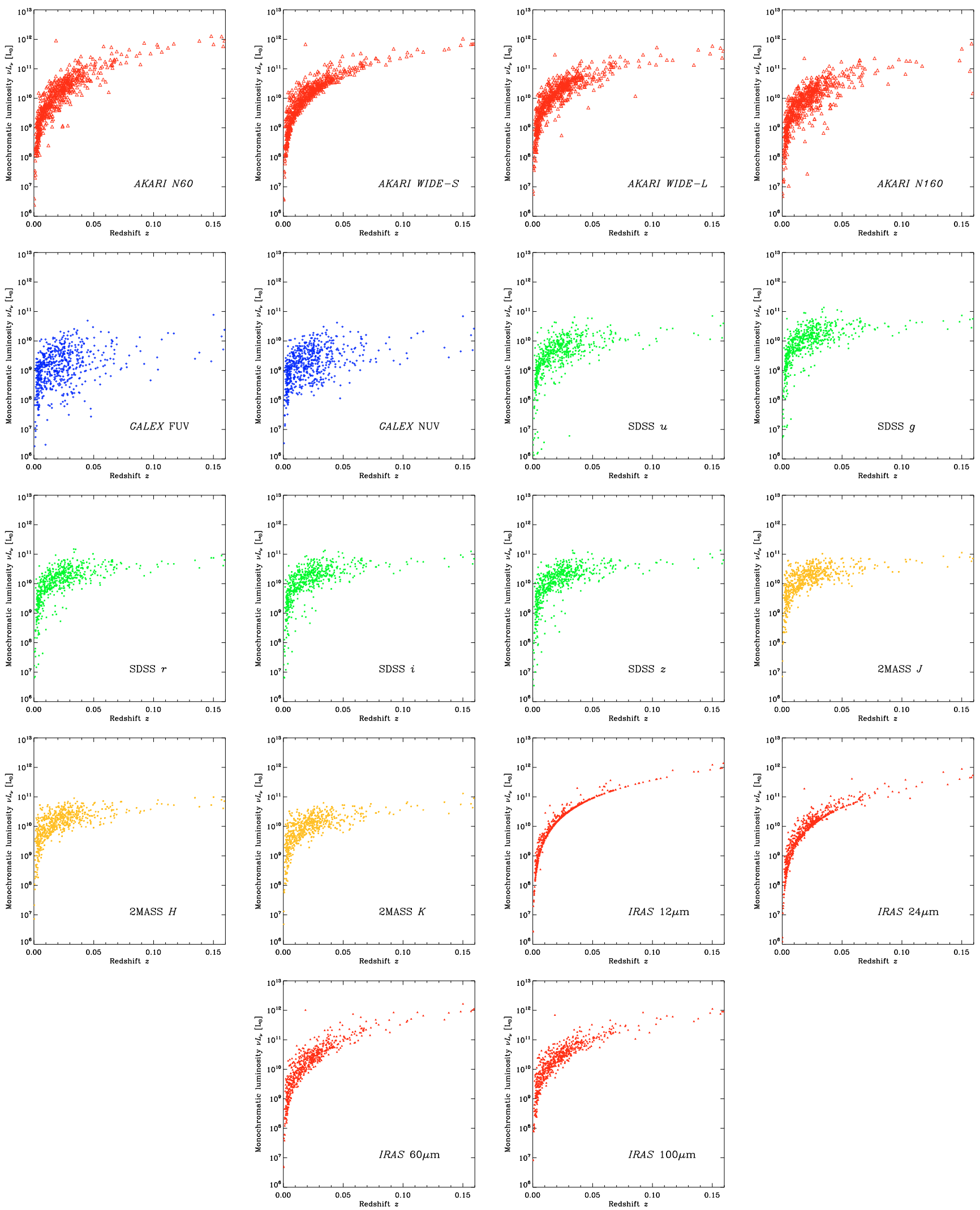

Fig. A.1. Luminosity vs. redshift diagrams for all the bands in the sample.

\section{References}

Bell, E. F., McIntosh, D. H., Katz, N., \& Weinberg, M. D. 2003, ApJS, 149, 289 Bell, E. F., Papovich, C., Wolf, C., et al. 2005, ApJ, 625, 23

Boissier, S., Gil de Paz, A., Boselli, A., et al. 2007, ApJS, 173, 524

Buat, V., Iglesias-Páramo, J., Seibert, M., et al. 2005, ApJ, 619, L51
Buat, V., Takeuchi, T. T., Iglesias-Páramo, J., et al. 2007a, ApJS, 173, 404 Buat, V., Marcillac, D., Burgarella, D., et al. 2007b, A\&A, 469, 19

Cardelli, J. A., Clayton, G. C., \& Mathis, J. S. 1989, ApJ, 345, 245 Chary, R., \& Elbaz, D. 2001, ApJ, 556, 562

Cortese, L., Boselli, A., Buat, V., et al. 2006, ApJ, 637, 242

Dale, D. A., \& Helou, G. 2002, ApJ, 576, 159 
Dale, D. A., Helou, G., Contursi, A., et al. 2001, ApJ, 549, 215

Dale, D. A., Bendo, G. J., Engelbracht, C. W., et al. 2005, ApJ, 633, 857

Daddi, E., Dickinson, M., Morrison, G., et al. 2007, ApJ, 670, 156

Dwek, E. 1998, ApJ, 501, 643

Dwek, E., \& Scalo, J. M. 1980, ApJ, 239, 193

Genzel, R., \& Cesarsky, C. J. 2000, ARA\&A, 38, 761

Gil de Paz, A., Boissier, S., Madore, B. F., et al. 2007, ApJS, 173, 185

Goldader, J. D., Meurer, G., Heckman, T. M., et al. 2002, ApJ, 568, 651

Helou, G., Khan, I. R., Malek, L., \& Boehmer, L. 1988, ApJS, 68, 151

Hirashita, H., Buat, V., \& Inoue, A. K. 2003, A\&A, 410, 83

Hirashita, H., Kaneda, H., Onaka, T., \& Suzuki, T. 2008, PASJ, 60, 477

Hopkins, A. M., \& Beacom, J. F. 2006, ApJ, 651, 142

Iglesias-Páramo, J., Buat, V., Takeuchi, T. T., et al. 2006, ApJS, 164, 38

Jarrett, T. H., Chester, T., Cutri, R., et al. 2000, AJ, 119, 2498

Jeong, W.-S., Nakagawa, T., Yamamura, I., et al. 2007, PASJ, 59, 429

Johnson, B. D., Schiminovich, D., Seibert, M., et al. 2007, ApJS, 173, 377

Kartaltepe, J. S., Sanders, D. B., Frayer, D. T., et al. 2009, ASP Conf. Ser., 408, 272

Kawada, M., Baba, H., Barthel, P. D., et al. 2007, PASJ, 59, 389

Kong, X., Charlot, S., Brinchmann, J., \& Fall, S. M. 2004, MNRAS, 349, 769

Kroupa, P. 2001, MNRAS, 322, 231

Martin, D. C., Fanson, J., Schiminovich, D., et al. 2005, ApJ, 619, L1

Martin, D. C., Wyder, T. K., Schiminovich, D., et al. 2007, ApJS, 173, 342

Meurer, G. R., Heckman, T. M., Leitherer, C., et al. 1995, AJ, 110, 2665

Meurer, G. R., Heckman, T. M., \& Calzetti, D. 1999, ApJ, 521, 64

Morrissey, P., Conrow, T., Barlow, T. A., et al. 2007, ApJS, 173, 682

Murakami, H., Baba, H., Barthel, P., et al. 2007, PASJ, 59, 369

Nozawa, T., Kozasa, T., Umeda, H., Maeda, K., \& Nomoto, K. 2003, ApJ, 598, 785

Onaka, T., Matsuhara, H., Wada, T., et al. 2007, PASJ, 59, S401

Pollo, A., Rybka, P., \& Takeuchi, T. T. 2010, A\&A, 514, A3

Neugebauer, G., Habing, H. J., van Duinen, R., et al. 1984, ApJ, 278, L1
Reddy, N. A., \& Steidel, C. C. 2009, ApJ, 692, 778

Reddy, N. A., Steidel, C. C., Pettini, M., et al. 2008, ApJS, 175, 48

Salim, S., Rich, R. M., Charlot, S., et al. 2007, ApJS, 173, 267

Salpeter, E. E. 1955, ApJ, 121, 161

Sanders, D. B., \& Mirabel, I. F. 1996, ARA\&A, 34, 749

Saunders, W., Sutherland, W. J., Maddox, S. J., et al. 2000, MNRAS, 317, 55

Schlegel, D. J., Finkbeiner, D. P., \& Davis, M. 1998, ApJ, 500, 525

Seibert, M., Martin, D. C., Heckman, T. M., et al. 2005, ApJ, 619, L55

Skrutskie, M. F., Cutri, R. M., Stiening, R., et al. 2006, AJ, 131, 1163

Soifer, B. T., Houck, J. R., \& Neugebauer, G. 1987, ARA\&A, 25, 187

Soifer, B. T., Helou, G., \& Werner, M. 2008, ARA\&A, 46, 201

Takeuchi, T. T., \& Ishii, T. T. 2004, ApJ, 604, 40

Takeuchi, T. T., Hirashita, H., Ohta, K., et al. 1999, PASP, 111, 288

Takeuchi, T. T., Ishii, T. T., Hirashita, H., et al. 2001a, PASJ, 53, 37

Takeuchi, T. T., Kawabe, R., Kohno, K., et al. 2001b, PASP, 113, 586

Takeuchi, T. T., Yoshikawa, K., \& Ishii, T. T. 2003a, ApJ, 587, L89

Takeuchi, T. T., Hirashita, H., Ishii, T. T., Hunt, L. K., \& Ferrara, A. 2003b, MNRAS, 343, 839

Takeuchi, T. T., Buat, V., \& Burgarella, D. 2005a, A\&A, 440, L17

Takeuchi, T. T., Buat, V., Iglesias-Páramo, J., Boselli, A., \& Burgarella, D. 2005b, A\&A, 432, 423

Takeuchi, T. T., Ishii, T. T., Nozawa, T., Kozasa, T., \& Hirashita, H. 2005c, MNRAS, 362, 592

Vergani, D., Zamorani, G., Lilly, S., et al. 2010, A\&A, 509, A42

Verma, A., Charmandaris, V., Klaas, U., Lutz, D., \& Haas, M. 2005, Space Sci. Rev., 119, 355

Yamamura, I., Fukuda, Y., \& Makiuti, S. 2008, AKARI/FIS all-sky Survey Bright Source Catalogue Version $\beta$-1 Release Note (Rev. 1)

Yamamura, I., et al. 2009, in AKARI, a light to illuminate the misty Universe, ed. T. Onaka, G. White, T. Nakagawa, I. Yamamura, ASP Conf. Ser., in press Yang, X., Mo, H. J., van den Bosch, F. C., et al. 2007, ApJ, 671, 153

Zheng, X. Z., Bell, E. F., Rix, H.-W., et al. 2006, ApJ, 640, 784 